\section{An Effect of X-Radiation on the 5 Potential of Colloidal Graphite}

IN their recent communication on the above subject $^{1}$, Prof. J. A. Crowther and H. Liebmann have discussed results obtained with graphite sols containing only a little ammonia.

A later paper ${ }^{2}$ describes an effect, which, if genuine, must be of importance in connexion with the biological effects of X-radiation. They find that on adding a "small quantity of surface-active material" to a sol of the above type, the sensitivity of the sol to X-rays is completely destroyed.

If this is the case, it is unlikely that the striking effect of X-rays upon the surface-charge of a colloid discovered by these authors can occur during the irradiation of cancerous animal tissue, since most living cells contain surface-active substances.

\section{IAN KEMP.}

70 Penns Lane,

Birmingham 24. Feb. 25 .

1 NATURE, 140, 28 (1937).

${ }^{2}$ Crowther, J. A., Liebmann, H., and Lane, T. B., Phil. Mag., 24, 654 (1937).

I AM very much aware of the fact that there is a very wide gulf between the simplicity of a graphite sol and the complexity of the intra-nuclear structures of living cells. If, from any unguarded expression in our paper, Mr. Kemp has been led to suppose that we imagined the gulf to have been crossed, he is wise to lodge a caveat. The curious effect of irradia. tion on the cataphoretic velocity observed by us in the case of colloidal graphite seems to be common to lyophobic sols, in general, and we have obtained it not only with amorphous carbon, both positive and negative, but also with gold, quartz, and ferric hydroxide.

The effect of irradiation on colloids of biological interest is now under investigation. Pending the results of these experiments, we should hesitate to draw general conclusions from the single observation to which Mr. Kemp directs attention, nor would we dogmatize as to possible applications to living cells. Too little is known about the chemistry of the cell and its nucleus. It is known, however, that biological changes are produced in the nucleus by doses of the order of magnitude which we are using in our experiments, and in view of the importance of the subject, any possible line of approach is worth exploring.

The University,

$$
\text { J. A. Crowther. }
$$

Reading.

March 5.

\section{Structure of Heavy Elementary Particles}

THE discovery of the heavy electron has recently led Bhabha ${ }^{1}$ and Heitler to discuss in detail Yukawa's suggestion to account for nuclear forces by the assumption that a heavy particle is surrounded by a heavy electron of mass $m$ during a fraction of time $q$.

It may be emphasized that this theory permits a very elementary treatment and allows us to obtain several consequences without extensive calculations. This is due to the fact that the emitted heavy electron, satisfying a wave equation of the type

$$
\square u-\varkappa_{-0}^{2} u=f(r) . \delta(r) \exp \left(\frac{2 \pi i}{h} W t\right), \varkappa_{0}=\frac{2 \pi m c}{h}
$$

is provided with an energy of the order of mass difference between proton and neutron, $W \sim \Delta M c^{2} \sim 10^{8}$ ev. (Taking the recoil into account: $W \sim \frac{1}{2} m c^{2} \frac{m}{M} \pm \Delta M ; m \geqslant \sqrt{2 M \Delta M .)}$ It behaves outside the proton (or neutron) like a free particle in the limiting relativistic case $W \ll m c^{2} ; u \sim \exp (-x r) / r$.

The theory meets with difficulties similar to those of electrodynamics, but it is not unlikely that the problem of light nuclei falls within its domain of applicability. If this is the case, we are led to the following conclusions :

(1) The theory gives for the degree of dissociation the value

$$
q=\frac{1}{1+\sqrt{1-\left(W / m c^{2}\right)^{2}}} \sim \frac{1}{2},
$$

which means that proton-proton forces are only slightly smaller than proton-neutron forces.

(2) The magneton of the dissociating heavy electron becomes

$$
\mu=\frac{h}{2 \pi} \cdot \frac{e c}{W},
$$

of the order of one ordinary Bohr magneton. Thus, only a small percentage of the dissociation can be directed by the spin of the heavy particle.

A detailed account of these considerations will be given in the Physical Review.

Cité Universitaire,

Guido BecK.

Fondation Hellénique,

45 Boulevard Jourdan, Paris (XIV).

March 1.

${ }^{1}$ NATURE, 141, 118 (1938).

\section{Methods of Estimating Mental Factors}

IN a recent letter ${ }^{1}$, Prof. G. H. Thomson has deduced an interesting relation between the equations which he and I have respectively proposed for estimating mental factors. It seems worth noting that this relation depends on a simple connexion between our estimates that previously $I$ had not realized. Let the test scores for any one person depend on his or her group and specific factors, $f_{0}$ and $f_{1}$, according to the (vector) equation :

$$
z=M f=M_{0} f_{0}+M_{1} f_{1} \text {. }
$$

From the identity $K=J(1-K)$, where $J \equiv$ $M_{0}^{\prime} M_{1}^{-2} M_{0}, \quad K \equiv M_{0}^{\prime} R^{-1} M_{0}, R \equiv M M^{\prime}=M_{0} M_{0}^{\prime}+$ $M_{1}^{2}$, it is easy to establish the reversible relation between our respective estimates for $f_{0}$,

$$
\hat{f}_{0}=K \check{f}_{0}, \quad \check{f}_{0}=K^{-1} \hat{f}_{0} .
$$

Thus as a (vector) statistic one estimate is equivalent to the other. Their different interpretation and properties might conveniently be summarized here by the formulæ :

$$
\begin{aligned}
& E\left\{\hat{f}_{0}\right\}=E\left\{f_{0}\right\}=0, E\left\{\left(\hat{f}_{0}-f_{0}\right)\left(\hat{f}_{0}-f_{0}\right)^{\prime}\right\}=1-K ; \\
& E_{1}\left\{\check{f}_{0}\right\}=f_{0}, E_{1}\left\{\left(\check{f}_{0}-f_{0}\right)\left(\check{f}_{0}-f_{0}\right)^{\prime}\right\}=J^{-1}=K^{-1}(1-K),
\end{aligned}
$$

where $E$ denotes averaging over all persons, $E_{1}$ over all possible sets of tests (comparable with the given set in regard to the amount of information on the group factors $f_{0}$ ). 